\title{
Stretching prior to resistance training promotes adaptations on the postsynaptic region in different myofiber types
}

\author{
Carolina dos Santos Jacob, Gabriela Klein Barbosa, Mariana Pasquini Rodrigues, Jurandyr Pimentel Neto, \\ Lara Caetano Rocha-Braga, Adriano Polican Ciena
}

Laboratory of Morphology and Physical Activity (LAMAF), São Paulo State University, Rio Claro, SP, Brazil

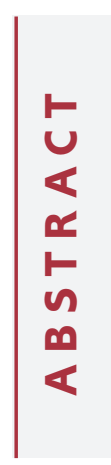

\begin{abstract}
The morphology of the neuromuscular junction adapts according to changes in its pattern of use, especially at the postsynaptic region according to the myofibrillar type and physical exercise. This investigation revealed the morphological adaptations of the postsynaptic region after static stretching, resistance training, and their association in adult male Wistar rats. We processed the soleus and plantaris muscles for histochemical (muscle fibers) and postsynaptic region imaging techniques. We observed muscle hypertrophy in both groups submitted to resistance training, even though the cross-section area is larger when there is no previous static stretching. The soleus postsynaptic region revealed higher compactness and fragmentation index in the combined exercise. The resistance training promoted higher adaptations in the postsynaptic area of plantaris; moreover, the previous static stretching decreased this area. In conclusion, the neuromuscular system's components responded according to the myofiber type even though it is the same physical exercise. Besides, static stretching (isolated or combined) plays a crucial role in neuromuscular adaptations.
\end{abstract}

Key words: Neuromuscular junction; motor endplate; muscle hypertrophy; static stretching; resistance training.

Correspondence: Adriano Polican Ciena, Ph.D., Laboratory of Morphology and Physical Activity (LAMAF), Institute of Biosciences (IB), Department of Physical Activity, São Paulo State University (UNESP), Avenue 24A, n 1515, 13506-900, Rio Claro, SP, Brazil. E-mail: adriano.ciena@unesp.br

Contributions: APC, CSJ, GKB, conceived and designed the research study; CSJ, GKB, MRP, performed the training protocols; APC, CSJ, GKB, JPN, LCR, analyzed data; CSJ, APC, wrote the manuscript. All authors read and approved the final manuscript.

Conflict of interest: The authors declare no conflict of interest.

Funding: This work was supported by São Paulo Research Foundation (FAPESP) (grant number\#2017/125251), in part by the Coordenação de Aperfeiçoamento de Pessoal de Nível Superior - Brazil (CAPES), Financing Code 001, and UNESP Rio Claro, Brazil, PROPG Edital 01/2022. 


\section{Introduction}

Exercise training promotes increased neuromuscular activity and demonstrates the plasticity of the neuromuscular junction (NMJ), resulting in improved endurance and resistance training in different ages. ${ }^{1,2}$ Moreover, the NMJ presents potential in postsynaptic component adaptations. The increase in acetylcholine receptors $(\mathrm{AChR})$ and endplate compactness, ensuring efficient communication between the pre and postsynaptic region and prolonged muscle fatigue..$^{3-5}$

Resistance training (RT) can promote chronic muscular adaptations in humans, and it is advocated as a fundamental exercise modality to improve muscle size and function development. ${ }^{6.7}$ These adaptations are predominantly due to increasing the muscle cross-section area (CSA) through the differentiation and proliferation of satellite cells by the release of inflammatory cytokines and growth factors. ${ }^{8}$ The manipulation of their variables (series, repetitions, frequency, overload, and rest interval) is directly proportional to the adaptive biological responses progress. ${ }^{9}$ Static stretching has been widely practiced among individuals of all physical activity levels, commonly prescribed during the warm-up of strength training. ${ }^{10,11}$ Furthermore, static stretching does not require much time or effort, has a low risk of injury in young adults, and has shown beneficial postural stability results. ${ }^{12,13}$ Evidence suggests that stretching performed before resistance training can directly and negatively influence strength production, number of repetitions, total volume, and muscle hypertrophy. ${ }^{14-16}$

It is essential to understand the possible effects in the postsynaptic region of the NMJ caused by static stretching and resistance training, especially in your combination. Furthermore, the composition of a muscle fiber type can be determined according to intrinsic and extrinsic responses such as genetics, hormones, aging, and type of exercise. ${ }^{17,18}$ The soleus muscle is located deep into the gastrocnemius muscle, and it is responsible for plantar flexion acting in jumping, walking, knee flexion, and like a peripheral heart in the return of venous blood. ${ }^{19,20}$ The plantaris is the vestigial remains of a muscle that originally continues the plantar aponeurosis as seen in some quadrupedal primates to aid in the prehension like the palmaris longus muscle. ${ }^{21}$ The plantaris muscle is also fixed in the superficial posterior compartment of the lower leg as an accessory soleus muscle. ${ }^{22}$ Although these muscles seem to work simultaneously, they present a different composition of fiber types. Soleus presents high content of Type I muscle fibers; besides, plantaris presents Type IIx (or IIb) muscle fiber predominance. ${ }^{23}$ Therefore, we investigated the postsynaptic region plasticity of soleus and plantaris muscle in 8 weeks of static stretching, resistance training, and their protocol combination in adult male Wistar rats.

\section{Materials and Methods}

\section{Animals}

Thirty-two 60-day-old male Wistar rats were divided into 4 groups ( $\mathrm{n}=8)$ : No-Training (NT); Resistance Training (RT); Stretching Training (ST); and Stretching-Resistance Training (SRT). During the experimental period, the animals were kept in cages $(33 \times 40 \times 16 \mathrm{~cm})(\mathrm{n}=4)$, under the conditions of temperature monitoring $\left(23 \pm 2{ }^{\circ} \mathrm{C}\right)$ and $12 \mathrm{~h}$ light/dark period, with food and water ad libitum. The Ethics Committee on the Use of Animals (CEUA) of the Biosciences Institute of the São Paulo State University (UNESP) approved this study (nº 09/2019).

\section{Stretching training protocol}

The ST and SRT groups performed an 8-week (24 sessions) stretching protocol (3 times/week), which consisted of using the non-quantified manual force applied to the plantar portion of the right posterior limb with the movement of dorsiflexion up to the range of motion limit of the talocrural joint. With the manual assistance of a specific investigator, the Wistar rats performed 10 movements of $30 \mathrm{~s}$ of static stretching, followed by a 30 -s interval resting in the neutral joint position. ${ }^{24}$ The same investigator executed all sessions. SRT group performed the stretching and immediately followed by resistance training in a vertical ladder.

\section{Resistance training protocol}

The Wistar rats of RT and SRT groups performed an 8-week (24 sessions) resistance training protocol (3 times/week ) in a vertical ladder $\left(110 \times 18 \mathrm{~cm}, 2 \mathrm{~cm}\right.$ grid, $80^{\circ}$ incline $)$. The sessions consisted of 4 to 9 progressive load climbs. They were allowed to rest for $120 \mathrm{~s}$ at the top of the ladder after each climb. ${ }^{25}$ The animals performed the first four climbs with $50 \%, 75 \%, 90 \%$, and $100 \%$ of their body mass additional loads fixed to the tail's proximal region. In the subsequent climbs, $30 \mathrm{~g}$ of the extra progressive load was added until the $9^{\text {th }}$ climb or exhaustion occurred. ${ }^{26}$ The Wistar rats performed the training protocols at the same time of the day across the experimental period.

\section{Histochemistry and morphometric analysis}

The soleus and plantaris muscle belly samples ( $n=5$ in each) of experimental groups were dissected and cryofixed, then transverse sections were made $(10 \mu \mathrm{m})$ (Cryostat HM 505 E, MICROM ${ }^{\mathrm{TM}}$, CA, USA) on microscope slides $(n=75)$. The histochemical reaction was used to differentiate the fiber types (type I and II in soleus / type I, IIa, and IIx in plantaris muscle). ${ }^{27}$

Twenty-five slides were incubated for $30 \mathrm{~min}$ at $37^{\circ} \mathrm{C}$ in a solution containing adenosine triphosphate (ATP - $10 \mathrm{mg}$ ) dissolved in three drops of distilled water, glycine $/ \mathrm{NaCl}_{2} / \mathrm{CaCl}_{2}$ buffer $(10 \mathrm{~mL})$, and $46.5 \mathrm{mg}$ of dithiothreitol for $30 \mathrm{~min}$ until reaching $\mathrm{pH}$ 9.4. For $\mathrm{pH} 4.3$ and 4.6 , the slides $(\mathrm{n}=25$ in each $\mathrm{pH})$ were preincubated in $0.1 \mathrm{M}$ sodium acetate buffer solution and $10 \mathrm{mM}$ ethylenediaminetetraacetic acid (EDTA) solution for $10 \mathrm{~min}$ at $4^{\circ} \mathrm{C}$. After, the slides were incubated in a solution containing ATP (10 mg), glycine $/ \mathrm{NaCl}_{2} / \mathrm{CaCl}_{2}$ buffer $(10 \mathrm{~mL})$, and $46.5 \mathrm{mg}$ of dithiothreitol for $30 \mathrm{~min}$. The final stage for all slides was incubation in $2 \%$ cobalt chloride for $7 \mathrm{~min}$, dehydration in a series of alcohol concentrations $(70 \%, 90 \%, 95 \%$ and $100 \%)$, then the slides were finished in xylol. ${ }^{28}$

The cross-section area (CSA) of type I and II of soleus myofibers and the CSA of type I, IIa, and IIx of plantaris myofibers ( $n=100 /$ fiber type/group) were observed using a $20 x$ objective lens with 10X ocular magnification. The images were obtained by a Zeiss ${ }^{\mathrm{TM}}$ Axioskop (Jena, Germany) light microscope. ${ }^{29}$ After, the morphometry was performed in each $\mathrm{pH}$ by the Image $\mathrm{JT}^{\mathrm{TM}}$ software (National Institutes of Health, Bethesda, MD, USA). Subsequently, we performed the normality test of the data and analyzed it using Kruskal-Wallis with Dunn's post-hoc test $(\mathrm{p}<0.05)$.

\section{Postsynaptic imaging - Tissue preparation}

The samples of soleus and plantaris muscle ( $n=3$ in each) of experimental groups were dissected and cryofixed in liquid nitrogen $\left(-196^{\circ} \mathrm{C}\right)$, then longitudinal sections $(100 \mu \mathrm{m}$ thickness $)$ were made (Cryostat HM 505 E, MICROMTM, CA, USA) to visualize the postsynaptic component of each NMJ. The sections were collected in silanized slides, pre-treated with $0.1 \%$ Triton-X solution, and washed $(3 \times 5 \mathrm{~min})$ in phosphate-buffered saline (PBS). The sections were incubated overnight at $4^{\circ} \mathrm{C}$ in a solution containing $\alpha$-bungarotoxin conjugated with rhodamine (BTX; Molecular 
Probes, Eugene, OR, USA; T-1175), diluted 1:600 in PBS containing $1 \%$ bovine serum albumin (BSA); washed in PBS before being coated with Prolong (Molecular Probes; P10144), coverslips applied and stored at $-20^{\circ} \mathrm{C}$ until analysis. ${ }^{30}$

\section{Postsynaptic region morphometric analysis}

The images $(n=20)$ for morphometric analysis were captured using a 100x objective lens with 10x ocular magnification by an Olympus BX61TM Fully Motorized Fluorescence Microscope (Shinjuku, Japan); equipped with a Fluorescence UIS2 optical system by Texas Red filter (Texas red excitation $596 \mathrm{~nm} / \mathrm{emission} 620$ $\mathrm{nm}$ ), obtained by a monochromatic camera Orca-Flash 2.8 (Hamamatsu, Japan) with the Software CellSens v.11 (Olympus ${ }^{\mathrm{TM}}$ ). With the ImageJTM Software (National Institutes of Health, Bethesda, Maryland, USA), it was measured the endplate area $\left(\mu \mathrm{m}^{2}\right)$ : the entire region composed of stained and unstained receptors; AChR area $\left(\mu \mathrm{m}^{2}\right)$ : the area composed of AChR; endplate perimeter $(\mu \mathrm{m})$ : the whole length covering the endplate composed of stained and unstained receptor; AChR perimeter $(\mu \mathrm{m})$ : the size covering around the AChR region; endplate diameter $(\mu \mathrm{m})$ : the maximum diameter formed by stained and unstained receptors; and the AChR clusters number (un) in the motor endplate (Figure 1).

The endplate compactness (\%) was measured for an accurate descriptor of receptors number within a given area, ${ }^{31}$ in other studies, we can find this index as a "dispersion": 32

\section{Compactness $=(\mathrm{AChR}$ area $) /($ endplate area $) \times 100$}

The endplate fragmentation index was measured, whereby an index of 0 means a solid plaque-like endplate, and an index close to 1 means a highly fragmented endplate: ${ }^{31}$

$$
\text { Fragmentation Index }=1-(1 /(\text { number of AChR clusters })
$$

We performed the Shapiro-Wilk's normality test $(\alpha=0.05)$ to verify the data's normal distribution, which presents a non-normal distribution; therefore, we analyzed it using Kruskal-Wallis with Dunn's post-hoc test $(\mathrm{p}<0.05)$.

\section{Results}

We reveal the alterations in the muscle fiber and postsynaptic region from soleus and plantaris of NT, ST, RT, and SRT groups.

\section{Cross-section area (CSA)}

In the histochemical analysis, the type I and II CSA from the soleus muscle and the type I, IIa, and IIx cross-section areas from plantaris of NT, ST, RT, and SRT groups were obtained (Table 1).

Soleus: the CSA of types I $(p<0.05)$ and II fibers $(p<0.0001)$ of the ST group were smaller compared to NT. The CSA of Type I fibers $(p<0.0001)$ of RT was larger compared to NT. Additionally, the CSA of Type II fibers $(\mathrm{p}<0.01)$ of RT was smaller than that found in NT. The CSA of types I $(p<0.05)$ and II $(p<0.001)$ fibers of SRT were larger than the NT group. The CSA of types I and II $(p<0.0001)$ fibers of SRT were larger than ST. Besides, the CSA of type I $(p<0.0001)$ fibers of SRT were smaller, and the Type II $(p<0.0001)$ fibers were larger than that found in the RT group.

Plantaris: the CSA of types I, IIa $(p<0.0001)$, and IIx $(p<0.001)$ fibers of ST were both larger compared to NT. The CSA of types I $(p<0.0001)$, IIa $(p<0.0001)$, and IIx $(p<0.0001)$ fibers of RT were both larger compared to NT. Furthermore, the CSA of Type I $(p<0.0001)$ and IIa $(p<0.0001)$ fibers of SRT were larger, and the type IIx fibers were smaller than those found in NT. In the SRT Group, the CSA of types I $(p<0.0001)$ and IIa $(p<0.01)$ fibers were larger, and type IIx $(p<0.0001)$ fibers were smaller compared to ST. Additionally, the CSA of types IIa $(p<0.001)$ and IIx (p 0.0001) fibers of SRT were both smaller, and the type I ( $p<0.0001)$ fibers were larger than that found in RT.

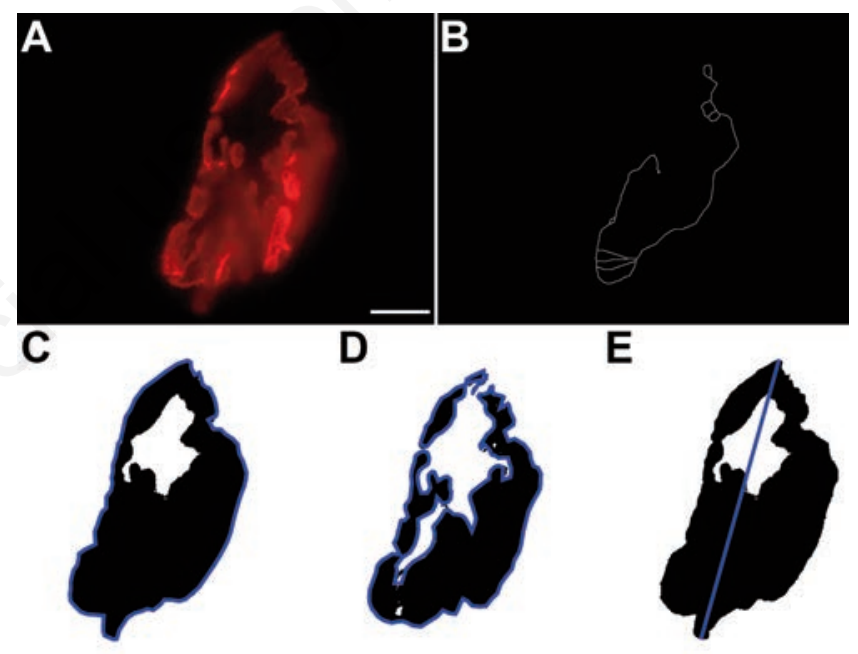

Figure 1. Postsynaptic region morphometry. (A) Original image of the postsynaptic region; scale bars: $10 \mu \mathrm{m}$. B) The example of AChR clusters measured. C) Binary image and the endplate perimeter and area measured. D) Binary image and the AChR perimeter and area measured. E) Binary image and the endplate diameter measured.

Table 1. Mean \pm standard deviation of cross-section area (CSA - $\mu^{2}$ ) of myofibers type I and II in the soleus muscle and I, IIa, IIx in the plantaris muscle of the No-Training (NT), Stretching Training (ST), Resistance Training (RT), and Stretching-Resistance Training (SRT) groups.

\begin{tabular}{|c|c|c|c|c|c|}
\hline \multirow{2}{*}{ Muscle } & \multirow[t]{2}{*}{ CSA } & \multicolumn{4}{|c|}{ Groups } \\
\hline & & NT & ST & RT & SRT \\
\hline \multirow[t]{2}{*}{ Soleus } & Type I & $3584.0 \pm 773.3$ & $3504.5 \pm 887.1$ & $4326.5 \pm 903.1^{\dagger, \text { 韦 }}$ & $3847.3 \pm 793.6$ \\
\hline & Type II & $3297.9 \pm 747.2$ & $2759.2 \pm 588.4^{\dagger}$ & $2892.4 \pm 354.4^{\S}$ & $3819.6 \pm 523.9$ \\
\hline \multirow[t]{3}{*}{ Plantaris } & Type I & $1132.1 \pm 187.2$ & $1247.3 \pm 356.6$ & $1769.9 \pm 466.1^{\ddagger}$ & $1801.2 \pm 339.5^{\ddagger}$ \\
\hline & Type IIa & $1207.3 \pm 219.8$ & $1594.0 \pm 331.1^{\ddagger}$ & $1918.9 \pm 419.1^{\ddagger}$ & $1791.7 \pm 334.6$, \\
\hline & Type IIx & $2763.7 \pm 832.0$ & $3266.4 \pm 843.1^{\dagger}$ & $3718.5 \pm 910.2^{\ddagger}$ & $2741.2 \pm 524.1^{\dagger,+}$ \\
\hline
\end{tabular}

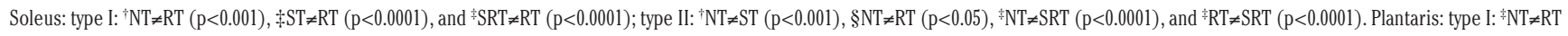
$(p<0.0001),{ }^{*} \mathrm{NT} \neq \mathrm{SRT}(\mathrm{p}<0.0001)$, and ${ }^{*} \mathrm{ST} \neq \mathrm{SRT}(\mathrm{p}<0.0001)$. Type IIa: ${ }^{*} \mathrm{NT} \neq \mathrm{ST}(\mathrm{p}<0.0001),{ }^{*} \mathrm{NT} \neq \mathrm{RT}(\mathrm{p}<0.0001),{ }^{\ddagger} \mathrm{NT} \neq \mathrm{SRT}(\mathrm{p}<0.0001)$, and ${ }^{\mathrm{s} T} \neq \mathrm{SRT}(\mathrm{p}<0.01) ;$ type IIx: ${ }^{*} \mathrm{NT} \neq \mathrm{RT}(\mathrm{p}<0.0001),{ }^{\dagger} \mathrm{NT} \neq \mathrm{ST}$ $(\mathrm{p}<0.001),{ }^{*} \mathrm{RT} \neq \mathrm{SRT}(\mathrm{p}<0.0001)$, and ${ }^{\top} \mathrm{ST} \neq \mathrm{SRT}(\mathrm{p}<0.001)$. 


\section{Postsynaptic region}

The area and perimeter of the endplate and AChR of soleus present smaller values in the ST group than NT (Figure 2). Moreover, in the ST group, the diameter, compactness, and number of AChR clusters of the endplate were smaller than that found in the NT group. The area and perimeter of the endplate and AChR of soleus were both smaller in the RT group compared to NT. Furthermore, in the RT group, the diameter, and compactness of the endplate were smaller; and the number of AChR clusters was higher than that found in the NT. The area and perimeter of the endplate and AChR of soleus were both smaller in the SRT group compared to NT. Besides, the endplate diameter and the number of AChR clusters of SRT were both smaller compared to NT. In SRT, the compactness endplate showed larger than NT. The area and perimeter of the endplate of SRT of soleus were smaller compared to ST. However, the AChR area and perimeter, and endplate compactness were larger in SRT than that found in the ST group. Furthermore, the endplate diameter and the number of AChR clusters of SRT showed minor values compared to ST.

The area of endplate and AChR of SRT was smaller than RT. The endplate perimeter and diameter of SRT were both smaller than that found in the RT. Besides, the AChR perimeter, the endplate compactness, and the number of AChR clusters of SRT were larger than RT. There was no statistical difference in the results of the postsynaptic region of the soleus (Figure 2). The AChR area and perimeter, and the area and perimeter of the plantaris muscle endplate were larger $(p<0.01)$ in the ST group than in the NT. The endplate diameter, compactness, and the number of AChR clusters of ST were larger than those found in the NT. The AChR area and perimeter, and the area and perimeter of the endplate were larger $(p<0.05)$ in RT than in the NT. Furthermore, the endplate diameter, compactness, and the number of AChR clusters $(p<0.01)$ of RT were both larger than NT. The endplate area of SRT of plantaris was smaller compared to NT. Additionally, the endplate perimeter and the AChR perimeter and area of SRT were larger than the NT; the endplate compactness $(p<0.01)$ was even larger. The endplate diameter and the number of AChR clusters of SRT were smaller compared to NT. The area $(p<0.01)$ and perimeter $(p<0.05)$ of the endplate, and the AChR area and perimeter $(\mathrm{p}<0.01)$ of plantaris were smaller in SRT than that found in the ST. Besides, the AChR area, the endplate compactness $(p<0.05)$, and the number of AChR clusters $(\mathrm{p}<0.01)$ of SRT were larger than ST. The endplate diam-
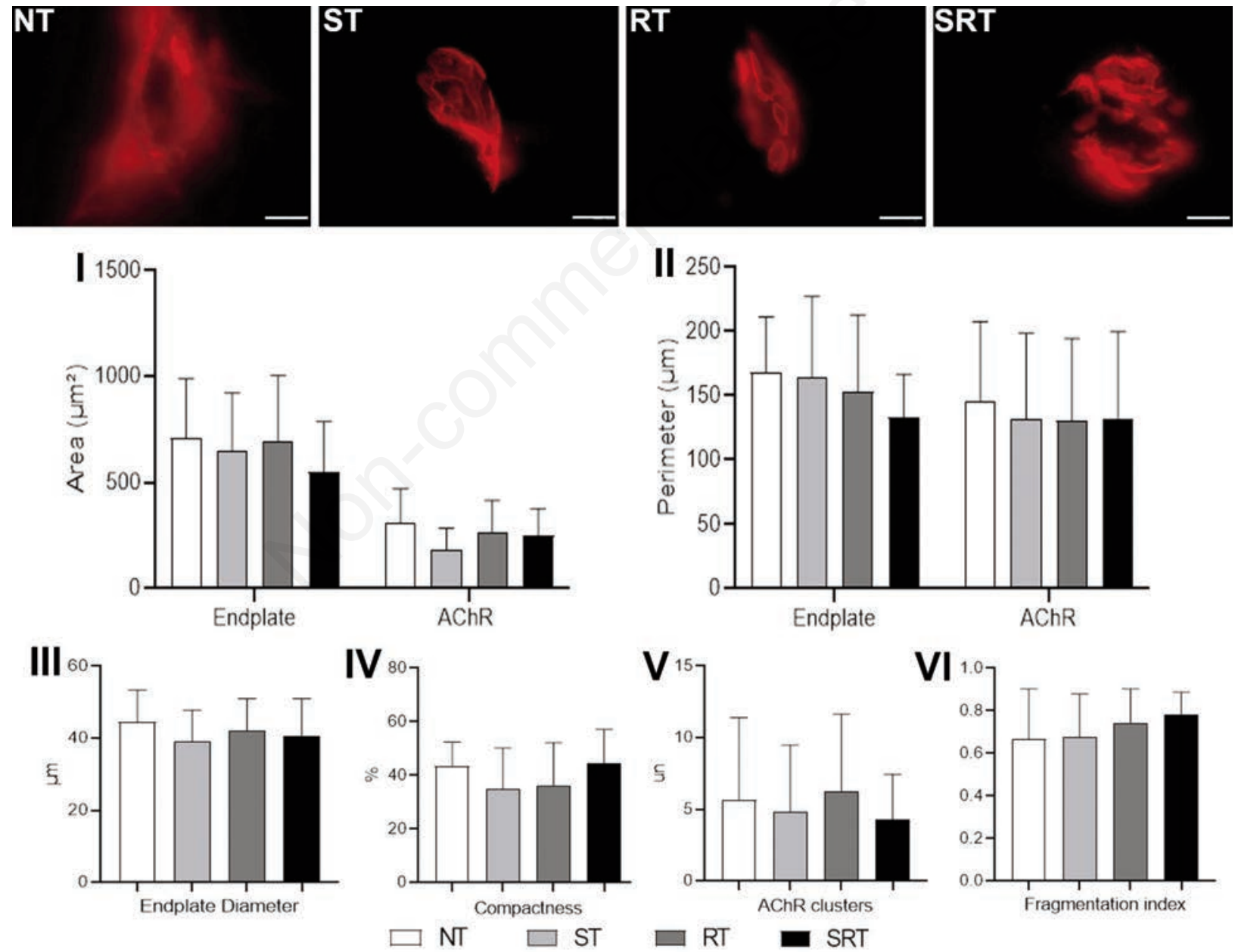
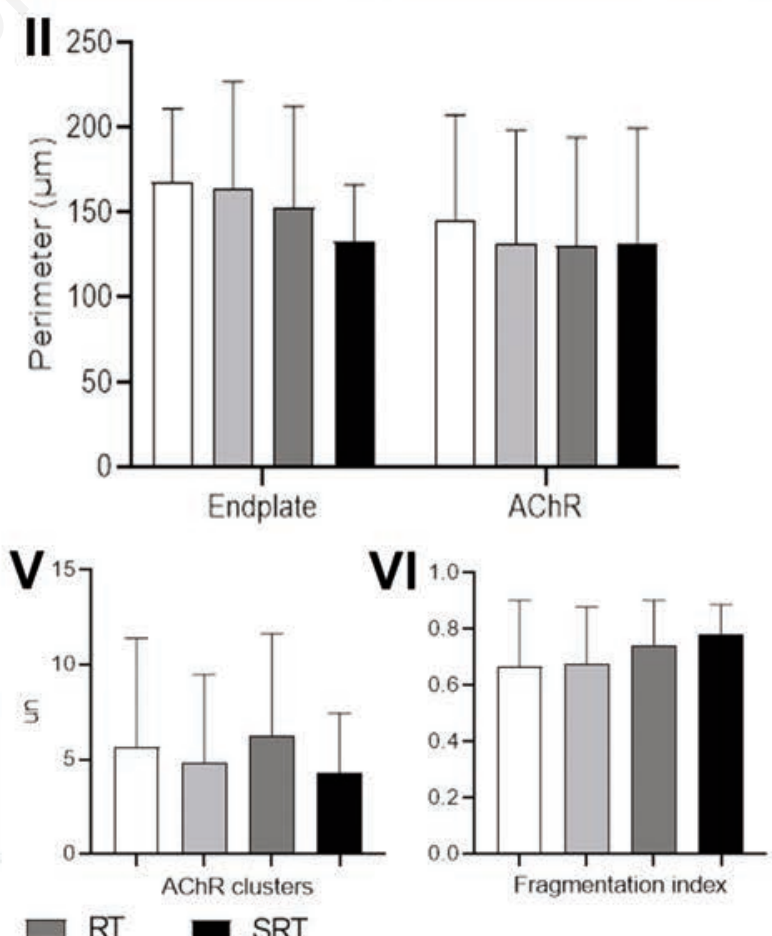

Figure 2. Postsynaptic AChR region of the soleus muscle of No-Training (NT), Stretching Training (ST), Resistance Training (RT), and Stretching-Resistance Training (SRT) groups; scale bars: $10 \mu \mathrm{m}$. I) Mean \pm standard deviation values of the endplate and AChR areas $\left(\mu \mathrm{m}^{2}\right)$. II) Mean \pm standard deviation values of the endplate and AChR perimeters $(\mu \mathrm{m})$. III) Mean \pm standard deviation values of endplate diameter $(\mu \mathrm{m})$. IV) Mean \pm standard deviation values of endplate compactness $(\%)$. V) Mean \pm standard deviation values of the number of AChR clusters (un). VI) Mean \pm standard deviation values of the fragmentation index. 
eter was smaller in SRT than in ST. In SRT, the AChR $(p<0.01)$ area and perimeter, and the area and perimeter of the endplate $(p<0.0001)$ were smaller compared to RT. Moreover, the endplate diameter of SRT was smaller than that found in RT $(\mathrm{p}<0.05)$. The endplate compactness and the number of AChR clusters in SRT were both larger than RT (Figure 3).

\section{Discussion}

These results suggest an increase in NMJ activity, specifically in the postsynaptic region after resistance training. ${ }^{33}$ Our recent finding revealed the effects of static stretching, resistance training, and their association with the myofibers CSA and postsynaptic region development. These findings are distinct between the muscles regarding the morphofunctional characteristics.

Regular resistance training is the main intervention to promote muscular adaptations, such as increased muscle mass, CSA,

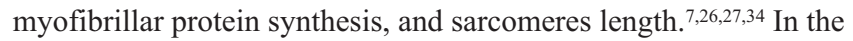
RT group, we observed hypertrophy of muscle fibers through the higher CSA of type I of soleus and type IIx myofibers of plantaris muscle. These adaptations in the muscle fibers architecture are directly associated with muscle strength production, where fibers with higher CSA values can develop higher muscle strength production due to the new sarcomeres' formation. ${ }^{35,36}$ Also, Allen et $a l .{ }^{37}$ suggested that muscle hypertrophy is correlated with the addition of new myonuclei through satellite cells' activity. A recent study by Rocha et al. ${ }^{29}$ showed soleus muscle hypertrophy in both fiber types after 24 sessions of resistance training in the vertical ladder which corroborates our results.

Regarding the static stretching response in the CSA of the muscle fibers, Peviani et al. ${ }^{38}$ demonstrated no difference in the soleus of experimental groups after 10 and 15 days of static stretching. In humans, Simpson et al. ${ }^{39}$ observed an increase in the lower limb's muscular thickness after 6 weeks of overloaded stretch training. These adaptations seem to occur according to stretching protocol intensity.

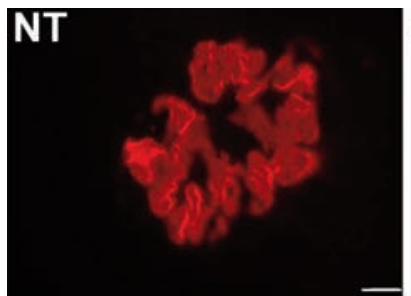

\section{ST}
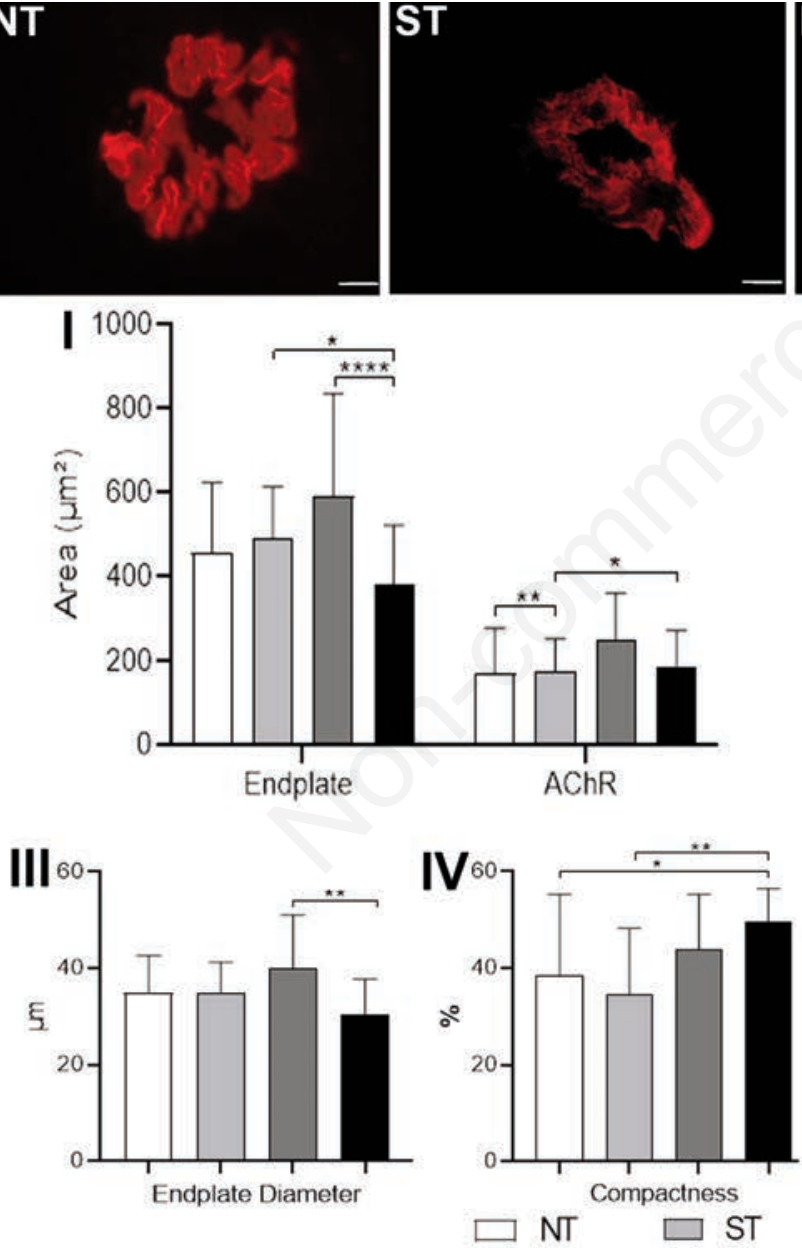

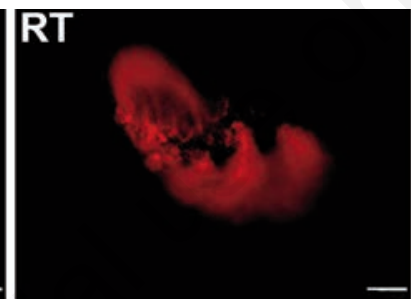

\section{SRT}

II
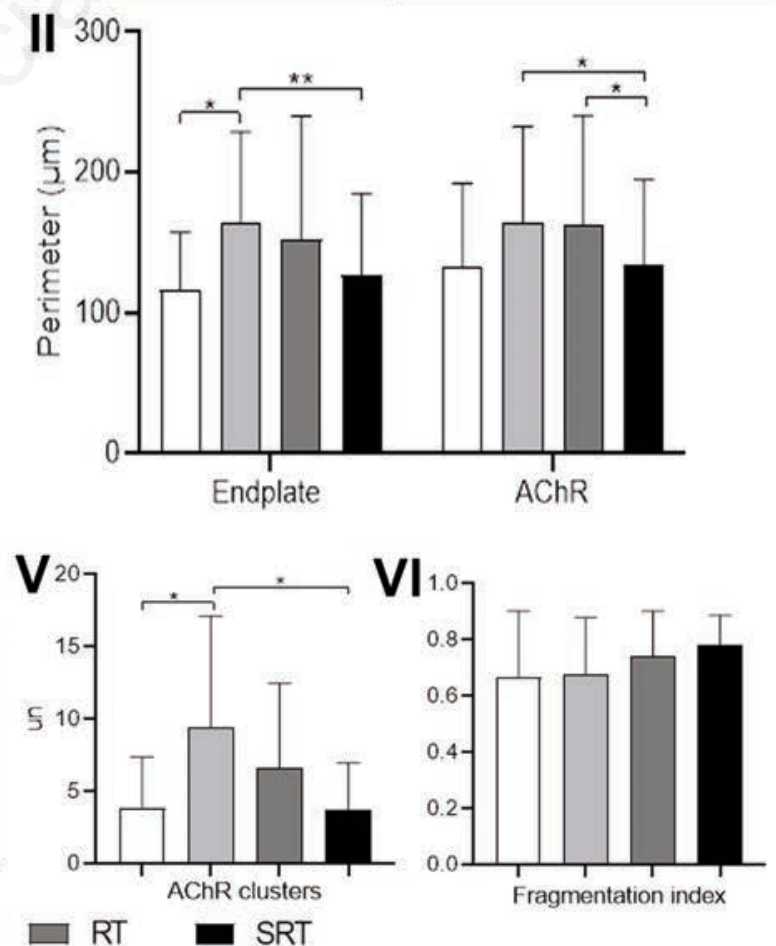

Figure 3. Postsynaptic AChR region of the plantaris muscle of No-Training (NT), Stretching Training (ST), Resistance Training (RT), and Stretching-Resistance Training (SRT) groups; scale bars: $10 \mu \mathrm{m}$. I) Mean \pm standard deviation values of the endplate and AChR areas $\left(\mu^{2}\right) ; * S T \neq$ SRT $(p<0.05) ;{ }^{* * * *} R T \neq$ SRT $(p<0.0001) ;{ }^{* *} N T \neq S T(p<0.01) ;{ }^{*} S T \neq$ SRT $(p<0.05)$. II $)$ Mean \pm standard deviation values of the endplate and AChR perimeters $(\mu \mathrm{m}) ;{ }^{*} \mathrm{NT} \neq \mathrm{ST}(\mathrm{p}<0.05) ;{ }^{* *} \mathrm{ST} \neq \mathrm{SRT}(\mathrm{p}<0.01) ;{ }^{*} \mathrm{ST} \neq \mathrm{S} \mathrm{RT}(\mathrm{p}<0.05) ;{ }^{*} \mathrm{RT} \neq \mathrm{SRT}(\mathrm{p}<0.05)$. III) Mean \pm standard deviation values of endplate diameter $(\mu \mathrm{m})$; ${ }^{* *} \mathrm{RT} \neq \mathrm{SRT}(\mathrm{p}<0.01)$. IV) Mean \pm standard deviation values of endplate compactness $(\%) ;{ }^{*} \mathrm{NT} \neq \mathrm{SRT}(\mathrm{p}<0.05) ;{ }^{*} \mathrm{ST} \neq \mathrm{S} \mathrm{RT}(\mathrm{p}<0.05)$. V) Mean \pm standard deviation values of the number of AChR clusters (un); ${ }^{*} \mathrm{NT} \neq \mathrm{ST}(\mathrm{p}<0.05) ;{ }^{*} \mathrm{ST} \neq \mathrm{SRT}(\mathrm{p}<0.05)$. VI) Mean \pm standard deviation values of the fragmentation index; ${ }^{* *} \mathrm{NT} \neq \mathrm{ST}(\mathrm{p}<0.01)$; $* *$ ST $\neq$ SRT $(\mathbf{p}<0.01)$. 
The stretching with overload does have a hypertrophic muscle effect, and changes in muscle size and architecture did not happen in the low-intensity stretch. ${ }^{40}$ Barbosa et al ${ }^{41}$ indicated that there was no increase in CSA of myofibers in the gastrocnemius muscle after 2 months of static stretching. Our results revealed a smaller CSA of myofiber compared to the NT group in soleus, differently in plantaris that presented muscle hypertrophy. This fact suggests that this static stretching protocol is enough and beneficial to adapt only in plantaris muscle fibers.

Similar to resistance training, manipulating variables in static stretching training seems to play a key role in neuromuscular adaptations. In soleus type I fibers, when static stretching is combined with resistance training, we can observe a non-significant increase in CSA. In contrast, the highest CSA value was considered in type II fibers after previous static stretching to resistance training. This indicates the hypertrophic response induced by stretching before resistance exercise seems to affect fast fibers, presumably due to the stretching of these fibers caused by static stretching and the tension generated through the load additional in resistance training.

The current literature suggests that low-intensity muscle stretching does not seem to respond significantly to muscle hypertrophy; however, high-intensity stretching can cause possible adaptations that are still limited. ${ }^{42}$ The practical implication of not performing the previous stretching is a shorter duration of the training sessions, improving adherence to exercise. ${ }^{43,44}$ In this study, changes in the postsynaptic region regarding static stretching and resistance training provided new data of plasticity in the peripheral nervous systems. We observed a reduction in the AChR and endplate perimeters, AChR and endplate areas, and consequently, the soleus postsynaptic region's compactness. Indeed, we observed an enlargement of the plantaris endplate and AChR area in ST and RT Group, interrupted when the stretching was performed before the resistance training (SRT Group). Morphological remodeling of the AChR area may be associated with desensitization during intense and repeated neural stimulation. ${ }^{33}$ This area's increase has a functional consequence of decreasing peripheral muscle fatigue during high-intensity muscle contractions. ${ }^{33}$ However, this area's reduction does not imply that the motor neuron's innervation in the muscle fibers is restricted considering that the postsynaptic potential usually has an amplitude that significantly exceeds the necessary to obtain a postsynaptic action potential. $^{45}$

Regarding resistance training on a vertical ladder, Deschenes et al..$^{31}$ presented data on young and elderly male Fischer 344 rats, where there were adaptations only in the postsynaptic components of the NMJ, as is evident in our RT data. Resistance training after static stretching (SRT group) resulted in higher endplate compactness than other experimental groups. The term compactness has been used as a descriptor of quantity receptors in the postsynaptic area; ${ }^{32}$ in other studies, we can find this index as a "dispersion", ${ }^{46}$ Deschenes et al. ${ }^{47}$ documented that compactness (or dispersion) is related to the intensity of exercise in which the resistance training increased soleus postsynaptic compactness, and in low intensity running, reduced endplate dispersion. The resistance training after stretching requires high neuromuscular activity, whereas the alterations in the distribution of synaptic receptors within the endplate region are induced by exercise type..$^{41}$ We can verify that the training intensity can influence the receptors' alterations in the postsynaptic region.

The endplate diameter showed changes similar to the endplate area, which are minor values in stretching groups. In Boehm et $a l .{ }^{48}$ work, each animal species has a different endplate diameter value. Indeed, Estrada-Bonilla et al. ${ }^{49}$ observed the NMJ morphometric characteristics of the upper limbs of healthy and diabetes mellitus-induced rats. These data differ from the lower limbs found in this study. There were also minor values in the diameter in conditions of diabetes compared to the control. ${ }^{49}$ However, the climbing training with and without an additional load in alternate sessions increases the endplate diameter and even the number of the biceps brachii muscle postsynaptic cleft. ${ }^{26}$

Regarding the fragmentation index, Prakash and Sieck ${ }^{50}$ found the effects of aging on NMJs morphological adaptations such as altered fragmentation. Although fragmentation may result from redistribution of the pre-synaptic components over a greater synaptic area, these alterations can also represent an increase in denervation and reinnervation, which may compromise motor innervation and contribute to muscle weakness. ${ }^{35}$ As a result, these exerciseinduced alterations need to be considered in training protocols or rehabilitation exercises to improve neuromuscular function. ${ }^{46}$

In conclusion, both groups submitted to resistance training showed a larger cross-section area of soleus myofibers, mainly without previous static stretching. The muscle-specific adaptation in the postsynaptic region occurs according to the type of exercise. However, the previous static stretching changes this remodeling by smaller AChR and endplate areas. Furthermore, static stretching contributes to the CSA muscle fiber development and can be proposed in functional and recovery exercise protocols. Despite the resistance training without static stretching is most beneficial for AChR remodeling and muscle hypertrophy, therefore the most recommended.

\section{Acknowledgments}

We thank Dr. Henrique Ferreira and his student's lab for their help with the fluorescence microscope.

\section{References}

1. Deschenes MR, Li S, Adan MA, Oh JJ, Ramsey HC. Muscle fibers and their synapses differentially adapt to aging and endurance training. Exp Gerontol 2018;106:183-91.

2. Deschenes MR. Adaptations of the neuromuscular junction to exercise training. Curr Opin Physiol 2019;10:10-6.

3. Deschenes MR, Maresh CM, Crivello JF, Armstrong LE, Kraemer WJ, Covault J. The effects of exercise training of different intensities on neuromuscular junction morphology. J Neurocytol 1993;22:603-15.

4. Fahim MA. Endurance exercise modulates neuromuscular junction of C57BL/6NNia aging mice. J Appl Physiol 1997;83:59-66.

5. Baehr LM, West DWD, Marcotte G, Marshall AG, Sousa LG, Baar K, et al. Age-related deficits in skeletal muscle recovery following disuse are associated with neuromuscular junction instability and ER stress, not impaired protein synthesis. Aging 2016;8:127-46.

6. Kraemer WJ, Ratamess NA. Fundamentals of resistance training: Progression and exercise prescription. Med Sci Sports Exerc 2004;36:674-88.

7. Schoenfeld BJ, Ratamess NA, Peterson MD, Contreras B, Sonmez GT, Alvar BA. Effects of different volume-equated resistance training loading strategies on muscular adaptations in well-trained men. J Strength Cond Res 2014;8:2909-18.

8. Marzetti E, Calvani R, Tosato M, Cesari M, Di Bari M, Cherubini A, et al. Physical activity and exercise as countermeasures to physical frailty and sarcopenia. Aging Clin Exp Res 2017;2935-42.

9. Qaisar R, Bhaskaran S, Van Remmen H. Muscle fiber type diversification during exercise and regeneration. Free Radic 
Biol Med 2016;98:56-67.

10. Kamonseki DH, Gonçalves GA, Yi LC, Lombardi Júnior I. Effect of stretching with and without muscle strengthening exercises for the foot and hip in patients with plantar fasciitis: A randomized controlled single-blind clinical trial. Man Ther 2016;23:76-82.

11. Sá MA, Matta TT, Carneiro SP, Araujo CO, Novaes JS, Oliveira LF. Acute effects of different methods of stretching and specific warm-ups on muscle architecture and strength performance. J Strength Cond Res 2015;30:2324-9.

12. Jamtvedt G, Hebert RD, Flottorp S, Odgaard-Jensen J, Havelsrud K, Narrat A et al. A pragmatic randomised trial of stretching before and after physical activity to prevent injury and soreness. Br J Sports Med 2010;44:1002-9.

13. Palmer TB, Agu-Udemba CC, Palmer BM. Acute effects of static stretching on passive stiffness and postural balance in healthy, elderly men. Phys Sportsmed 2018;46:78-86.

14. Barroso R, Tricoli V, Gil SS, Ugrinowitsch C, Roschel H. Maximal strength, number of repetitions, and total volume are differently affected by static-, ballistic-, and proprioceptive neuromuscular facilitation stretching. J Strength Cond Res 2012;26:2432-7.

15. Simão R, Lemos A, Salles B, Leite T, Oliveira E, Rhea M, et al. The influence of strength, flexibility, and simultaneous training on flexibility and strength gains. J Strength Cond Res 2011;25:1333-8.

16. Moriggi Júnior R, Berton R, Souza TMF, Chacon-Mikahil MPT, Cavaglieri CR. Effect of the flexibility training performed immediately before resistance training on muscle hypertrophy, maximum strength and flexibility. Eur J Appl Physiol 2017;117:767-74.

17. Cristea A, Qaisar R, Edlund PK, Lindblad J, Bengtsson E, Larsson L. Effects of aging and gender on the spatial organization of nuclei in single human skeletal muscle cells. Aging Cell 2010;9:685-97.

18. Maffei M, Longa E, Qaisar R, Agoni V, Desaphy JF, Camerino DC, et al. Actin sliding velocity on pure myosin isoforms from hindlimb unloaded mice. Acta Physiol 2014;212:316-29.

19. Joshi SS, Joshi SD, Kishve PS. Feathered fibres of human soleus muscle. Int J Morphol 2010;28:239-42.

20. Kawano MM, Ambar G, Oliveira BIR, Boer MC, Cardoso APRG, Cardoso JR. Influence of the gastrocnemius muscle on the sit-and-reach test assessed by angular kinematic analysis. Braz J Phys Ther 2010;14:10-5.

21. Keith A. The history of the human foot and its bearing on orthopaedic practice. J Bone Jt Surg 1929;11:10-32.

22. Kwinter DM, Lagrew JP, Kretzer J, Lawrence C, Malik D, Mater M, et al. Unilateral double plantaris muscle: a rare anatomical variation. Int J Morphol 2010;28:1097-9.

23. DeNies MS, Johnson J, Maliphol AB, Bruno M, Kim A, Rizvi A, et al. Diet-induced obesity alters skeletal muscle fiber types of male but not female mice. Physiol Rep 2014;2:e00204.

24. Gianelo MCS, Polizzelo JC, Chesca D, Mattiello-Sverzut AC. Three days of intermittent stretching after muscle disuse alters the proteins involved in force transmission in muscle fibers in weanling rats. Braz J Med Biol Res 2016;49:1-8.

25. Hornberger TA, Farrar RP. Physiological hypertrophy of the FHL muscle following 8 weeks of progressive resistance exercise in the rat. Can J Appl Physiol 2004;29:16-31.

26. Pimentel Neto J, Rocha LC, Jacob CS, Barbosa GK, Ciena, AP. Postsynaptic cleft density changes with combined exercise protocols in an experimental model of muscular hypertrophy. Eur J Histochem 2021;65:3274

27. Rocha LC, Pimentel Neto J, Sant'Ana JS, Jacob CS, Barbosa GK, Krause Neto W, et al. Repercussions on sarcomeres of the myotendinous junction and the myofibrillar type adaptations in response to different trainings on vertical ladder. Microsc Res Tech 2020;83:1190-7.

28. Brooke MH, Kaiser KK. The use and abuse of muscle histochemistry. Ann N Y Acad Sci 1974;228:121-44.

29. Rocha LC, Jacob CS, Barbosa GK, Pimentel Neto J, Krause Neto W, Gama EF, et al. Remodeling of the skeletal muscle and postsynaptic component after short-term joint immobilization and aquatic training. Histochem Cell Biol 2020;154:621-8.

30. Krause Neto W, Silva WA, Ciena AP, Anaruma CA, Gama EF. Divergent effects of resistance training and anabolic steroid on the postsynaptic region of different skeletal muscles of aged rats. Exp Gerontol 2017;98:80-90.

31. Deschenes MR, Sherman EG, Roby MA, Glass EK, Harris MB. Effects of resistance training on neuromuscular junctions of young and aged muscles featuring recruitment patterns. J Neurosci Res 2015;93:504-13.

32. Jones RA, Reich CD, Dissanayake KN, Kristmundsdottir F, Findlater GS, Ribchester RR et al. NMJ-morph reveals principal components of synaptic morphology influencing structurefunction relationships at the neuromuscular junction. Open Biol 2016;6:160240.

33. Deschenes MR, Judelson DA, Kraemer WJ, Meskaitis VJ, Volek JS, Nindl BC, et al. Effects of resistance training on neuromuscular junction morphology. Muscle Nerve 2020;23:1576-81.

34. Burd NA, Holwerda AM, Selby KC, West DWD, Staples AW, Cain NE, et al. Resistance exercise volume affects myofibrillar protein synthesis and anabolic signalling molecule phosphorylation in young men. J Physiol 2010;588:3119-30.

35. Zamparo P, Minetti AE, Di Prampero PE. Interplay among the changes of muscle strength, cross-sectional area and maximal explosive power: Theory and facts. Eur J Appl Physiol 2002;88:193-202.

36. Suchomel TJ, Nimphius S, Bellon CR, Stone MH. The Importance of Muscular Strength: Training Considerations. Sports Med 2018;48:765-85.

37. Allen DL, Roy RR, Edgerton VR. Myonuclear domains in muscle adaptation and disease. Muscle Nerve 1999;22:135060.

38. Peviani SM, Guzzoni V, Pinheiro-Dardis CM, Silva YP, Fioravante ACR, Sagawa AH, et al. Regulation of extracellular matrix elements and sarcomerogenesis in response to different periods of passive stretching in the soleus muscle of rats. Sci Rep 2018;8:9010.

39. Simpson CL, Kim BDH, Bourcet MR, Jones GR, Jakobi JM. Stretch training induces unequal adaptation in muscle fascicles and thickness in medial and lateral gastrocnemii. Scand J Med Sci Sports 2017;27:1597-604.

40. Nunes JP, Schoenfeld BJ, Najamura M, Ribeiro AS, Cunha PM, Cyrino ES. Does stretch training induce muscle hypertrophy in humans? A review of the literature. Clin Physiol Funct Imaging 2020;40:148-56.

41. Barbosa GK, Jacob CS, Rodrigues MP, Rocha LC, Pimentel Neto J, Ciena AP. Morphological changes in the motor endplate and in the belly muscle induced by previous static stretching to the climbing protocol. Microsc Microanal 2021;23:1-9.

42. Shrier I. Does stretching improve performance? A systematic and critical review of the literature. Clin J Sport Med 2004; 14:267-73.

43. Hoare E, Stavreski B, Jennings GL, Kingwell BA. Exploring motivation and barriers to physical activity among active and inactive Australian adults. Sports (Basel) 2017;5:1-8.

44. Nuzzo JL. The case for retiring flexibility as a major compo- 
nent of physical fitness. Sports Med 201950:853-70.

45. Deschenes MR, Hurst TE, Ramser AE, Sherman EG Presynaptic to postsynaptic relationships of the neuromuscular junction are held constant across age and muscle fiber type. Dev Neurobiol 2013;73:744-53.

46. Deschenes MR, Tufs HL, Oh J, Li S, Noronha AL, Adan MA. Effects of exercise training on neuromuscular junctions and their active zones in young and aged muscles. Neurobiol Aging 2020;95:1-8

47. Deschenes MR, Maresh CM, Crivello JF, Armstrong LE, Kraemer WJ, Covault J. The effects of exercise training of different intensities on neuromuscular junction morphology. J
Neurocytol 1993;22:603-15.

48. Boehm I, Alhindi A, Leite AS, Logie C, Gibbs A, Murray O, et al. Comparative anatomy of the mammalian neuromuscular junction. J Anat 2020;237:827-36.

49. Estrada-Bonilla YC, Castro PATS, Luna GLF, Souza ABA, Santos GS, Salvinia TF et al. Reaching task performance is associated to neuromuscular junction adaptations in rats with induced diabetes mellitus. Braz J Med Biol Res 2020;53:e8763.

50. Prakash YS, Sieck GC. Age-related remodeling of neuromuscular junctions on type-identified diaphragm fibers. Muscle Nerve 1998;21:887-95.

Received for publication: 29 October 2021. Accepted for publication: 1 February 2021.

This work is licensed under a Creative Commons Attribution-NonCommercial 4.0 International License (CC BY-NC 4.0).

CCopyright: the Author(s), 2022

Licensee PAGEPress, Italy

European Journal of Histochemistry 2022; 66:3356

doi:10.4081/ejh.2022.3356 